\title{
Development and implementation of a novel child life protocol to enhance psychosocial support for pediatric awake craniotomies: technical note
}

\author{
*Michael M. McDowell, MD, ${ }^{1,2}$ Daniela Ortega Peraza, MS, CCLS, ${ }^{3}$ and Taylor J. Abel, MD1,2,4 \\ 1'Division of Neurological Surgery, UPMC Children's Hospital of Pittsburgh; ${ }^{2}$ Department of Neurological Surgery, University of \\ Pittsburgh; ${ }^{3}$ Department of Child Life, UPMC Children's Hospital of Pittsburgh; and ${ }^{4}$ Department of Bioengineering, University of \\ Pittsburgh, Pennsylvania
}

\begin{abstract}
Awake craniotomies are a crucial tool for identifying eloquent cortex, but significant limitations frequently related to patient tolerance have limited their applicability in pediatric cases. The authors describe a comprehensive, longitudinal protocol developed in collaboration with a certified child life specialist (CCLS) in order to enhance patient experiences and develop resiliency related to the intraoperative portion of cases. This protocol includes preoperative conditioning, intraoperative support, and postoperative positive reinforcement and debriefing. A unique coping plan is developed for each prospective patient. With appropriate support, awake craniotomy may be applicable in a wider array of preadolescent and adolescent patients than has previously been possible. Future prospective studies are needed to validate this approach.
\end{abstract}

https://thejns.org/doi/abs/10.3171/2019.11.FOCUS19774

KEYWORDS pediatric; awake craniotomy; child life; psychosocial; support

$\mathrm{A}$ WAKE craniotomies are an important tool to mitigate the risks of postoperative neurological deficits in resective neurosurgery that involves eloquent language and or motor cortices. Whereas the procedure is well documented in adult cohorts with favorable surgical results, there are only scattered case reports and series in the pediatric literature. ${ }^{16,21}$ The existing pediatric literature demonstrates that awake craniotomy can play a crucial role in the safe resection of brain lesions in close proximity to language and motor cortex. ${ }^{3}$ Unfortunately, published results of awake craniotomy in younger children (age $<10$ years) are uncommon, perhaps given the common perspective that eloquent functions will recover or reorganize after ithe njury in young children. Despite this commonly held view, the literature and common practice demonstrate otherwise; there are many examples of children with a history of early injury to eloquent cortex (e.g., hemispherotomy) who do not demonstrate full recovery. Thus, developing and refining methods for eloquent cortical mapping in young children is an important priority.
Awake craniotomies in young children can be challenging given the psychological stress of undergoing an awake procedure. Cooperation with language and motor mapping tasks during this stress is crucial. For this reason, it has previously been suggested that children under the age of 10 years who require language mapping should undergo chronic electrode implantation for extraoperative mapping. ${ }^{20}$ However, successful awake craniotomies have been performed in children younger than 10 years, and to our knowledge in children as young as 6 years. ${ }^{14}$ The ability to ensure that pediatric patients psychologically tolerate the open craniotomy process is perhaps the greatest limitation in the application of this technique in the preadolescent and even adolescent age group.

In this paper, we describe the development and implementation of a unique child life protocol to increase the success of awake craniotomy in pediatric neurosurgery patients. It is well documented that psychosocial support by the involved providers is critical in the process of patient satisfaction and tolerance of the procedure, ${ }^{23}$

ABBREVIATIONS CCLS = certified child life specialist.

SUBMITTED September 27, 2019. ACCEPTED November 13, 2019.

INCLUDE WHEN CITING DOI: 10.3171/2019.11.FOCUS19774.

* M.M.M. and D.O.P. contributed equally to this work. 
and there have been reports regarding the use of preconditioning with the supervision of a psychiatrist in pediatric patients. ${ }^{19}$ However, to date there is no systematic, longitudinal approach to providing this support in pediatric patients. ${ }^{23}$ We provide our novel protocol driven by collaboration with a certified child life specialist (CCLS) dedicated to ensuring young patients' comfort and compliance during awake craniotomies.

\section{Methods}

We sought to develop and implement a novel child life protocol designed to enhance the patient experience and compliance surrounding awake craniotomy as well as strengthening the patient's ability to tolerate the process. The protocol was developed by the child life service (D.O.P. in conjunction with the senior author [T.J.A.]). Our protocol is divided into preoperative, intraoperative, and postoperative phases (Fig. 1).

\section{Preoperative Phase}

The CCLS reaches out to the patient's caregivers once an awake craniotomy has been scheduled at our institution. An in-person meeting is offered, but if declined, a conversation is scheduled by telephone. The child life program and available services are discussed, followed by time to provide preparation and answer questions and concerns. ${ }^{6}$ This allows for the patient and caregivers to express their questions and understanding of the surgery and the hospital stay. During these conversations several misconceptions regarding surgery and the hospital may arise, and the CCLS can address the concerns and provide education. ${ }^{5}$ Part of the conversation is getting to know the patient's hospitalization background and whether it has been a positive or negative experience so that it can be addressed, and reassuring the patient and family of the staff's commitment to meet all their needs in the best possible way.

There are patients who learn best in a hands-on experience, and a tour of the facilities and operating rooms is offered for those interested. This would also allow for the hospital to be familiar and for the patient and their family to know what to expect on the day of surgery. During a hospital tour the CCLS may have a medical play session with the patient to familiarize him or her with medical equipment and see it in a nonthreatening way. Photos of the operating room and setup for an awake craniotomy are available for review and discussion. These can be reviewed during the tour, or at home in the form of a preparation booklet to be reviewed with parental supervision. ${ }^{15}$ The photos and preparation booklets include descriptions in a child-friendly vocabulary and also explain the sensory experience at each step. Specific experiences include the focal sensory blockade using local anesthetic injections, minimal hair shaving, Mayfield pin placement, and the approximate location and extent of linear scalp incisions.

Based on the conversations with the patient and caregivers, an individualized coping plan for all phases of the hospitalization is developed with input from the patient and family. There is a need for individualized coping plans because every patient has different coping needs and interests. Customizing the plan allows the patient to feel more comfortable in the hospital, and it gives them control by the CCLS providing choices of coping methods that are designed with the patient's interests in mind. The creation and implementation of the coping plan is geared to the hospitalization because it gives the patient control of how to positively manage their needs when things get stressful and difficult. ${ }^{5}$ Throughout this process rapport is developed between the patient and CCLS, which is essential for the day of the surgery.

\section{Intraoperative Phase}

On the day of surgery, the CCLS meets the patient and family in the preoperative area to address questions and provide support for anxiety. The patient is taken to the operating room with the CCLS present to provide support, answer last-minute questions, and activate the coping plan as needed until sedation has taken effect. Later, the CCLS is present prior to wakening the patient intraoperatively. The CCLS's presence during the start of anesthesia is critical, because $40 \%-60 \%$ of children will display significant emotional and behavior stress in the immediate preoperative period, with a similar number reporting high anxiety specifically at the point of induction among children ages 3-12 years. ${ }^{8,13}$ Special care is taken to assess the environment of the patient, including what is visible. Care is taken to ensure that disturbing visuals such as blood, radiographic or intraoperative pictures, and vital signs are not visible. Additional pillows and blankets are used to ensure the comfort of the patients and that there is adequate body covering - and are critical especially in adolescent development for avoiding embarrassment over the exposure of genitalia. Once the patient is awake the coping plan is implemented as needed while positive emotional reinforcement is used to maintain patient compliance. Intraoperative mapping is tailored to the age and maturity of the patient. For patients most likely to benefit from this protocol, mapping tasks include reading (when possible), speaking, and singing. The CCLS remains visible and present until after the readministration of sedation.

\section{Postoperative Phase}

The postoperative period is critical to ensure that the experience is framed in as positive terms as possible, particularly in cases in which future awake procedures may be indicated. A dedicated time for "debriefing" in order to process the experience is necessary to ensure that patients have an opportunity to express their feelings from the surgery and related to having successfully (or unsuccessfully) complied with the awake portion of the case. Open-ended questions pertaining to their anxieties preoperatively, their discomfort and perceptions postoperatively, and their experiences intraoperatively are asked with ample pauses to allow for free association by the patient. In the case that the patient has limited verbal capabilities to express themselves, nonverbal methods of self-expression can be used. Nonverbal methods can include play, writing, art, music, etc. Patient experiences should be validated and any attempt at awake craniotomy should receive aggressive positive reinforcement by the entire medical staff as well as the CCLS. Memories are variable in quantity, clarity, and 


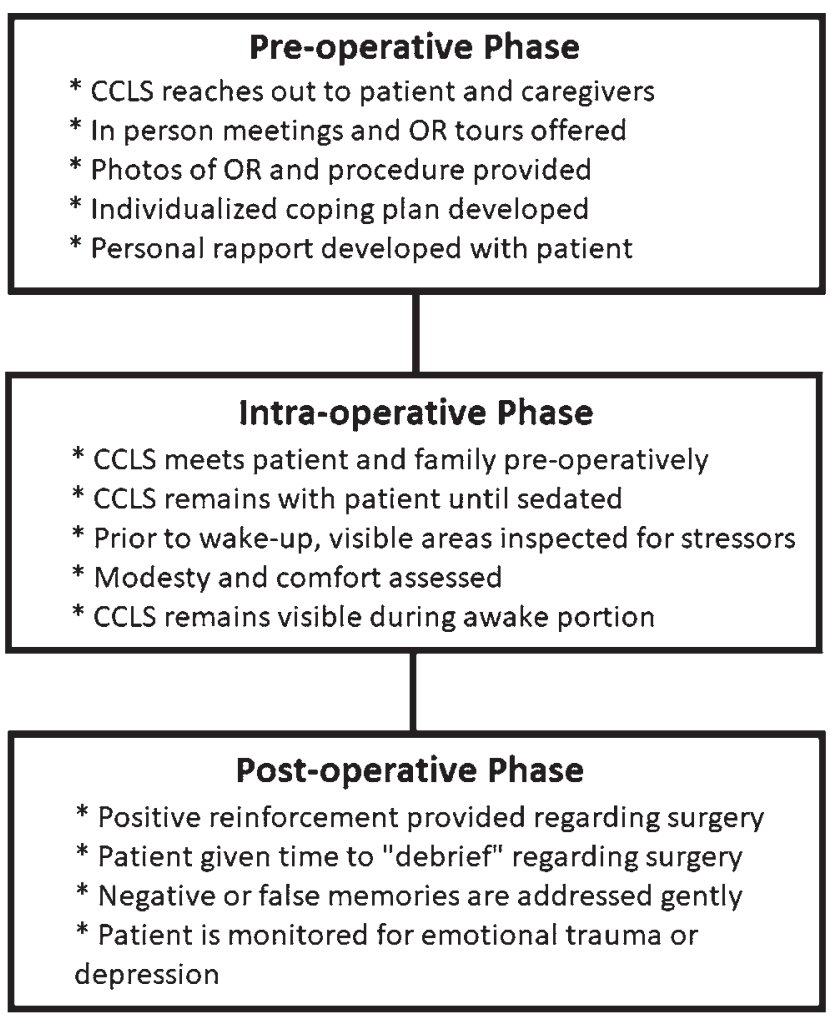

FIG. 1. Diagram depicting the phases of CCLS-driven patient support for awake craniotomy cases. $\mathrm{OR}=$ operating room.

basis in reality..$^{19}$ Any negative memories not based on real events should be gently addressed, but typically the recollections are positive. Any signs of emotional trauma or depression should be identified and addressed by an appropriate provider, although this has not yet been routinely the case. Standard posttraumatic stress disorder questions should be inserted into the flow of conversation without being obvious, if possible, in order to avoid feelings of selfconsciousness. These questions relate to disturbing encounters, feelings of stress when reminded of the past experience, avoidance of situations or activities that remind the patient of the past experience, feelings of dissociation or irritation, and difficulty with concentration.

\section{Clinical Experience}

To date, 2 patients have undergone surgery using the targeted approach described above, with both having surgery successfully completed as planned and recovering without permanent neurological deficit. One patient reported positive experiences in the perioperative period and the other reported having no clear recollection of the experience. Neither patient demonstrated evidence of posttraumatic stress disorder. However, both cases have been performed in only the last few months and thus long-term outcomes remain to be studied. One patient had no complications and the other suffered an intraoperative seizure at the time of closure. This case is described as follows.

This is a 16-year-old girl with Li-Fraumeni syndrome and an incidental nonenhancing intrinsic lesion of the left
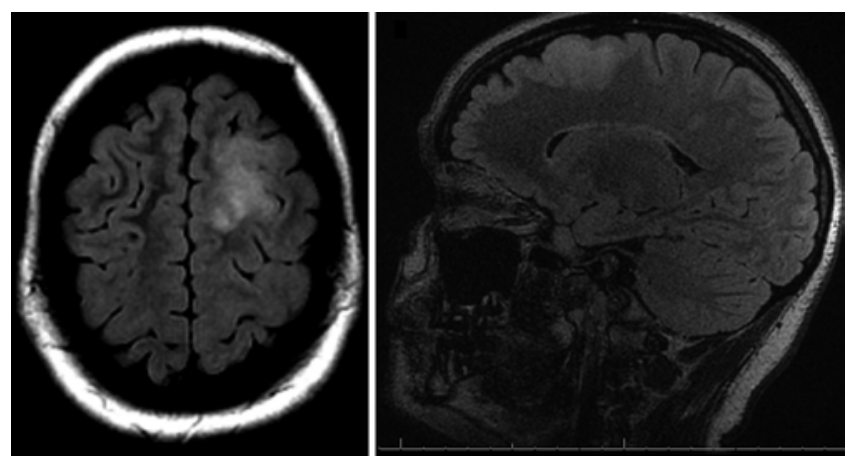

FIG. 2. Axial (left) and sagittal (right) preoperative FLAIR MRI sequences demonstrating a nonenhancing intrinsic lesion.

frontal lobe (Fig. 2). The patient was asymptomatic at the time of presentation except for a history of a febrile seizure at the age of 5 years. She was offered an awake left craniotomy using the child life protocol. Using image guidance after sedation, local anesthetic, and pinning, a left frontal craniotomy was performed via a linear hemicoronal incision. The patient was awakened without difficulty and the tumor was removed using cortical stimulation mapping, which the patient was able to perform easily with the support of a CCLS. At the end of the resection, the patient suffered a tonic-clonic seizure requiring intubation. An intraoperative MRI sequence was obtained (Fig. 3) confirming tumor removal, and the wound was closed in standard fashion. Pathology was consistent with a low-grade glioma. Postoperatively the patient demonstrated some transient speech delay but no other focal deficits. The patient did not recall the procedure or perioperative events.

\section{Discussion}

We demonstrate in this technical report our center's development and implementation of a child life protocol to decrease stress and increase compliance for pediatric awake craniotomy patients. Through careful psychological buttressing with this child life-driven protocol, we propose to increase pediatric awake craniotomy compliance and, potentially, outcomes. Adult series have been predominantly favorable, with enhanced outcomes in terms of neurological preservation, survival benefit, and greater tumor resection frequently being cited. ${ }^{4,10,21}$

Of 50 cases in which patients underwent awake neurosurgical procedures that were reviewed by Lohkamp et al., all but 4 cases were age 10 years or older and the 2 youngest documented children were 8 years old. ${ }^{16}$ Most reported cases of awake craniotomy in pediatric patients have been in adolescents, with a median age of 15 years. Fifty-two percent of cases were craniotomies for lesion within eloquent areas, $32 \%$ were for epilepsy surgery, and $16 \%$ were for deep brain stimulation. Age variances between these 3 categories were not assessed, but anecdotally an 8-year-old patient tolerated the awake process, in contrast to a 13-yearold patient who did not, suggesting that the magnitude of the surgery may not be so limiting as the psychology of the individual as well as possibly the preoperative conditioning conducted..$^{3,19}$ Inability to tolerate the awake phase of 


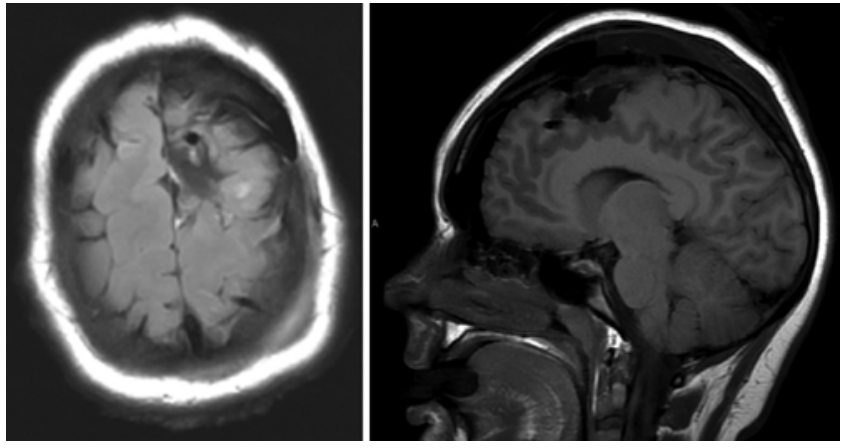

FIG. 3. Axial (left) and sagittal (right) postoperative FLAIR MRI sequences demonstrating complete resection.

the craniotomy is the cause of half of discontinuations. In total, 2 of 42 patients undergoing lesional resection or epilepsy surgery (4.8\%) suffered permanent new neurological deficits postoperatively. ${ }^{16}$

In extremely young patients awake craniotomy may not be a reasonable option given the low likelihood of compliance. For this reason, some have advocated for extraoperative mapping with invasive electrodes (e.g., subdural grids). ${ }^{20}$ Others have argued that awake craniotomies may be unnecessary given the concordance between cortical stimulation results and extraoperative stimulation mapping. ${ }^{18}$ However, much of the literature suggests that passive mapping methodologies are sensitive, but lack the specificity of cortical stimulation mapping. ${ }^{17}$ The specificity of passive mapping modalities may be further decreased in the pediatric population given developmental changes in cortical language maps., ${ }^{7,911,17,24}$ Therefore, the specificity of cortical stimulation mapping over passive mapping techniques may increase the amount of tumor (or other lesion) that can be safely resected. Given the risks of extraoperative mapping with invasive electrodes, pediatric awake craniotomy may be a relatively low-risk method for localizing eloquent cortex to maximize safe resection, and comes with the benefits of not requiring multiple procedures and potentially decreased morbidity from lead placement. pl, $^{12,25}$

Although not reported to date, there exist concerns related to long-term psychological trauma in addition to intraoperative compliance. ${ }^{16}$ Conditioning patients preoperatively as well as reinforcing the positive nature of successful tolerance of the protocol may reduce the likelihood of posttraumatic stress through the enhancement of patient resilience. ${ }^{22}$ In a study of 7 pediatric patients considered for awake craniotomy, Riquin et al. found that all 7 were eligible. ${ }^{19}$ Two of the patients had a high level of preoperative anxiety on neuropsychological assessment, but nonetheless tolerated the procedure well with appropriate conditioning.

CCLSs are an essential part of the multidisciplinary team in pediatric hospitals. Their jobs entail meeting the patient and his or her family's psychosocial needs. A CCLS has a strong background in child development and takes courses related to meeting specific needs in a pediatric setting, i.e., the hospitalized child, child development, death and bereavement, working with children with spe- cial needs, play, medical terminology, family systems, and many more. Beyond the course requirements to become a CCLS, one must have a minimum of a bachelor's degree, 600 hours of clinical experience, and must pass the child life professional accreditation examination. These are the requirements set by the Association of Child Life Professionals. CCLSs are the staff that can prepare for and explain the hospital experience to children in an age- and developmentally appropriate way. A CCLS's role is to address the psychosocial needs of both patients and families. These needs include emotional support, preparation for surgery and other procedures, and both identifying and facilitating coping strategies that are age and developmentally appropriate.

For patients who are undergoing awake craniotomies, we believe that a standardized process establishes a nonthreatening advocate to promote vocalization of the needs, frustrations, concerns, and questions that both patients and families may have but may not wish to directly address with the treating providers. The child life service provides a longitudinal, in-house outlet for patients to work through their emotions both before and after surgery, and the involvement of a child life specialist ensures that a friendly and familiar face is always present during the awake portions of surgery. Each patient's coping plan is developed with the patient and family in conjunction with their assigned CCLS. This plan is individualized to meet the specific needs and interests of each patient and includes components directed at the preoperative, intraoperative, and postoperative periods. Common requests include dedicated conversation time, choosing music for the operating room as well as the patient's room, pet therapy, and having favorite books on hand for reading.

\section{Future Directions}

Having demonstrated the feasibility of the protocol in early patients, we will seek to prospectively validate the generalizability and success of CCLS-supported awake craniotomies in pediatric patients. Patients eligible for awake craniotomy will be approached for enrollment. Compliance will be tracked in addition to postoperative outcomes and patient perceptions of the experience. Those who do not elect to have an awake craniotomy will serve as a control group.

\section{References}

1. Arya R, Wilson JA, Fujiwara H, Vannest J, Byars AW, Rozhkov L, et al: Electrocorticographic high-gamma modulation with passive listening paradigm for pediatric extraoperative language mapping. Epilepsia 59:792-801, 2018

2. Aungaroon G, Zea Vera A, Horn PS, Byars AW, Greiner HM, Tenney JR, et al: After-discharges and seizures during pediatric extra-operative electrical cortical stimulation functional brain mapping: Incidence, thresholds, and determinants. Clin Neurophysiol 128:2078-2086, 2017

3. Balogun JA, Khan OH, Taylor M, Dirks P, Der T, Snead III $\mathrm{OC}$, et al: Pediatric awake craniotomy and intra-operative stimulation mapping. J Clin Neurosci 21:1891-1894, 2014

4. Boetto J, Bertram L, Moulinié G, Herbet G, Moritz-Gasser S, Duffau H: Low rate of intraoperative seizures during awake craniotomy in a prospective cohort with 374 supratentorial 
brain lesions: electrocorticography is not mandatory. World Neurosurg 84:1838-1844, 2015

5. Boles J: Preparing children and families for procedures or surgery. Pediatr Nurs 42:147-149, 2016

6. Brewer S, Gleditsch SL, Syblik D, Tietjens ME, Vacik HW: Pediatric anxiety: child life intervention in day surgery. $\mathbf{J}$ Pediatr Nurs 21:13-22, 2006

7. Chou N, Serafini S, Muh CR: Cortical language areas and plasticity in pediatric patients with epilepsy: a review. Pediatr Neurol 78:3-12, 2018

8. Davidson AJ, Shrivastava PP, Jamsen K, Huang GH, Czarnecki C, Gibson MA, et al: Risk factors for anxiety at induction of anesthesia in children: a prospective cohort study. Paediatr Anaesth 16:919-927, 2006

9. Devous MD Sr, Altuna D, Furl N, Cooper W, Gabbert G, Ngai WT, et al: Maturation of speech and language functional neuroanatomy in pediatric normal controls. J Speech Lang Hear Res 49:856-866, 2006

10. Hervey-Jumper SL, Li J, Lau D, Molinaro AM, Perry DW, Meng L, et al: Awake craniotomy to maximize glioma resection: methods and technical nuances over a 27 -year period. J Neurosurg 123:325-339, 2015

11. Hyslop A, Duchowny M: Electrical stimulation mapping in children. Seizure [epub ahead of print], 2019

12. Jain P, Whitney R, Strantzas S, McCoy B, Ochi A, Otsubo H, et al: Intra-operative cortical motor mapping using subdural grid electrodes in children undergoing epilepsy surgery evaluation and comparison with the conventional extra-operative motor mapping. Clin Neurophysiol 129:2642-2649, 2018

13. Kain ZN, Caldwell-Andrews A, Wang SM: Psychological preparation of the parent and pediatric surgical patient. Anesthesiol Clin North America 20:29-44, 2002

14. Klimek M, Verbrugge SJ, Roubos S, van der Most E, Vincent AJ, Klein J: Awake craniotomy for glioblastoma in a 9-yearold child. Anaesthesia 59:607-609, 2004

15. Lerwick JL: Psychosocial implications of pediatric surgical hospitalization. Semin Pediatr Surg 22:129-133, 2013

16. Lohkamp LN, Mottolese C, Szathmari A, Huguet L, Beuriat PA, Christofori I, et al: Awake brain surgery in children-review of the literature and state-of-the-art. Childs Nerv Syst 35:2071-2077, 2019

17. Miller KJ, Abel TJ, Hebb AO, Ojemann JG: Rapid online language mapping with electrocorticography. J Neurosurg Pediatr 7:482-490, 2011

18. Papanicolaou AC, Rezaie R, Narayana S, Choudhri AF, Wheless JW, Castillo EM, et al: Is it time to replace the
Wada test and put awake craniotomy to sleep? Epilepsia 55:629-632, 2014

19. Riquin E, Dinomais M, Malka J, Lehousse T, Duverger P, Menei $P$, et al: Psychiatric and psychologic impact of surgery while awake in children for resection of brain tumors. World Neurosurg 102:400-405, 2017

20. Sanai N, Mirzadeh Z, Berger MS: Functional outcome after language mapping for glioma resection. N Engl J Med 358:18-27, 2008

21. Serletis D, Bernstein M: Prospective study of awake craniotomy used routinely and nonselectively for supratentorial tumors. J Neurosurg 107:1-6, 2007

22. Vyas KJ, Fesperman SF, Nebeker BJ, Gerard SK, Boyd ND, Delaney EM, et al: Preventing PTSD and depression and reducing health care costs in the military: a call for building resilience among service members. Mil Med 181:1240-1247, 2016

23. Wahab SS, Grundy PL, Weidmann C: Patient experience and satisfaction with awake craniotomy for brain tumours. Br J Neurosurg 25:606-613, 2011

24. Westmacott R, McAndrews MP, deVeber G: Language representation following left MCA stroke in children and adults: an fMRI study. Can J Neurol Sci 44:483-497, 2017

25. Yan H, Katz JS, Anderson M, Mansouri A, Remick M, Ibrahim GM, et al: Method of invasive monitoring in epilepsy surgery and seizure freedom and morbidity: a systematic review. Epilepsia 60:1960-1972, 2019

\section{Disclosures}

Dr. Abel is a consultant with Monteris.

\section{Author Contributions}

Conception and design: all authors. Acquisition of data: all authors. Analysis and interpretation of data: all authors.

Drafting the article: all authors. Critically revising the article: all authors. Reviewed submitted version of manuscript: all authors. Administrative/technical/material support: Abel.

\section{Correspondence}

Taylor J. Abel: University of Pittsburgh Medical Center, Pittsburgh,PA. abeltj@upmc.edu. 